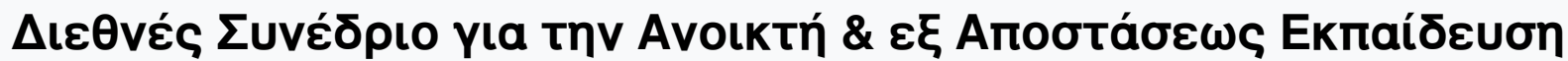

Tóp. 6, Ap. 2B (2011)

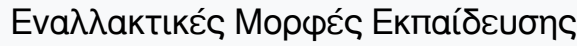

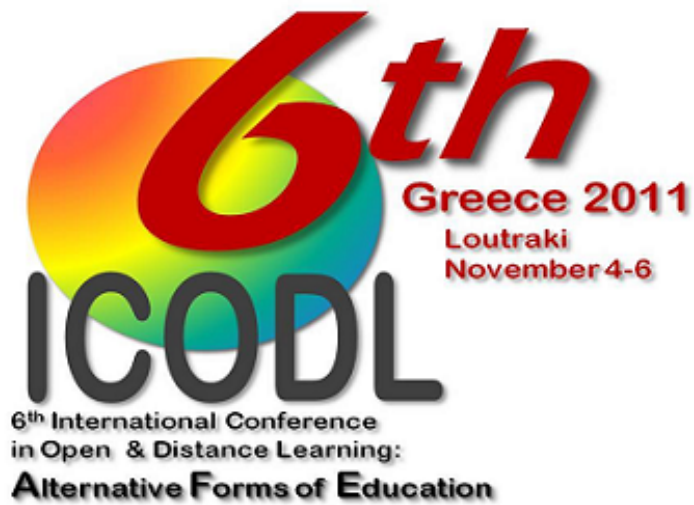

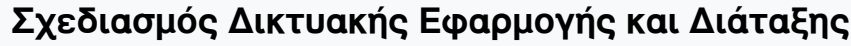

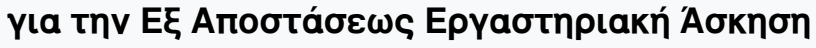

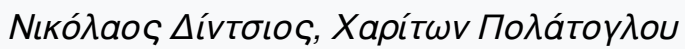

doi: $10.12681 /$ icodl. 669

TOMOE B

PART / MEPOE B 


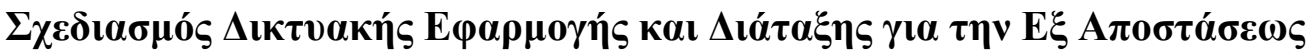

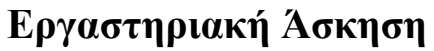

\section{Design of a Web Application and its Set Up for Distance Laboratory Exercise}

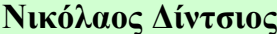

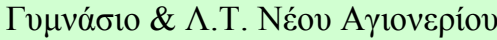 \\ $\mathrm{K} \alpha \theta \eta \gamma \eta \tau \eta \dot{\varsigma} \mathrm{B} / \theta \mu \mathrm{\alpha} \alpha \mathrm{E \kappa} \pi / \sigma \eta \varsigma$

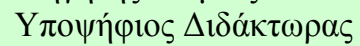 \\ nikos.dintsios@gmail.com
}

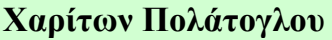

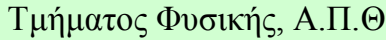

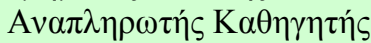 \\ hariton@auth.gr
}

\begin{abstract}
It is a common belief that real experiments are very crucial in education. Several times though, there is a lack of time in performing such experiments in laboratories. To face with this problem simulations have been developed using the internet making possible for students to visualize an experiment through the net. The next step beyond simulation would be to conduct real experiments using the internet. In this project we are developing a hardware and a software setup in order to achieve this goal and make experimental e-Learning reality. The project will be accomplished when students will participate and several conclusions will arise.
\end{abstract}

\section{Пері́ $\eta \psi \eta$}

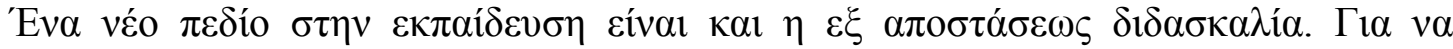

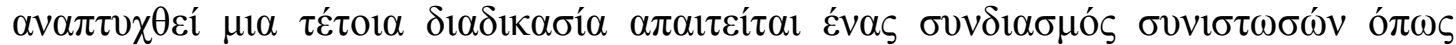

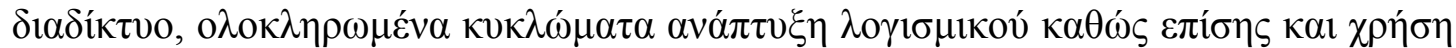

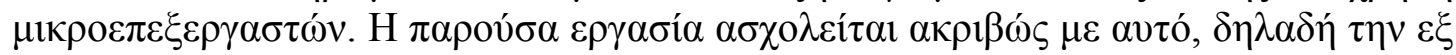

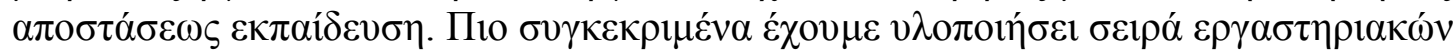

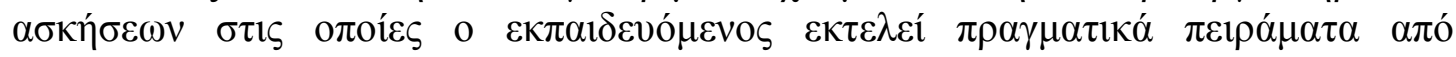

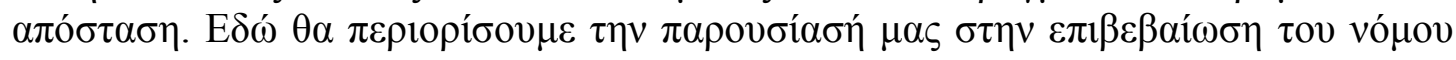

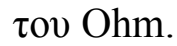

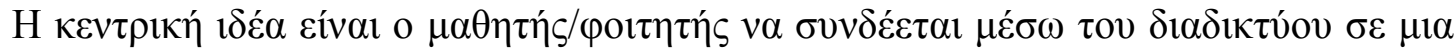

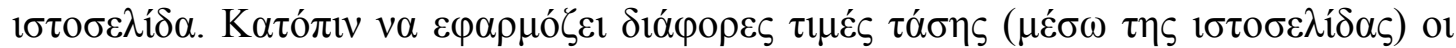

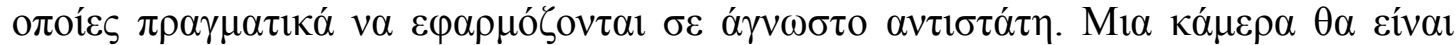

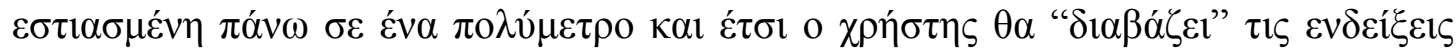

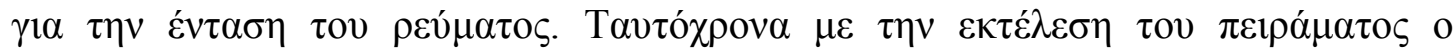

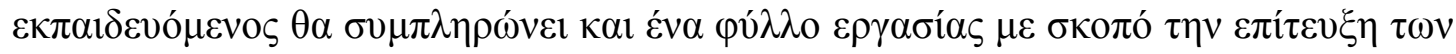

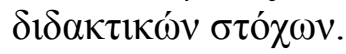

\section{Key-words: distance learning, real experiment}

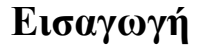

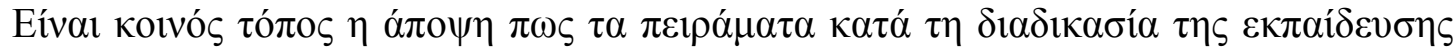

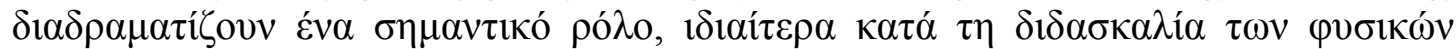

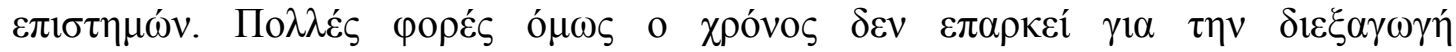

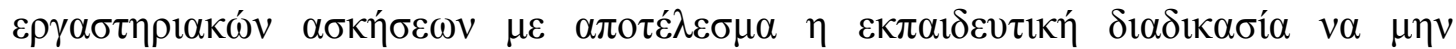

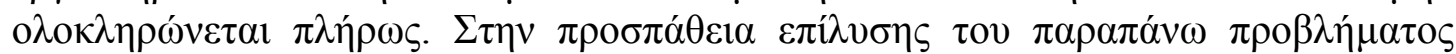




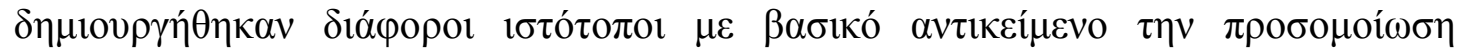

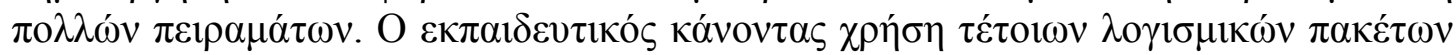

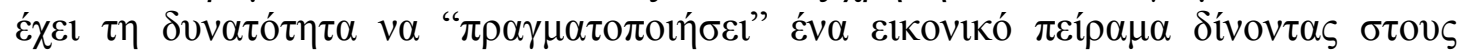

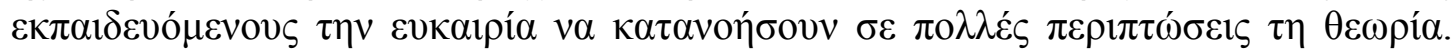

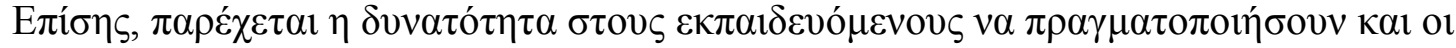

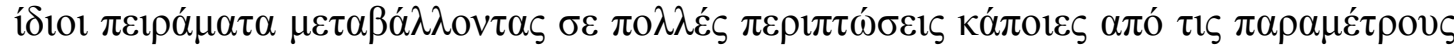

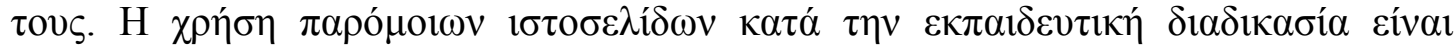

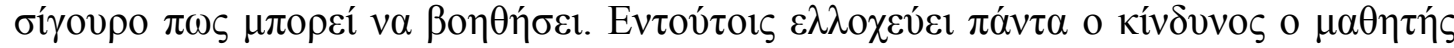

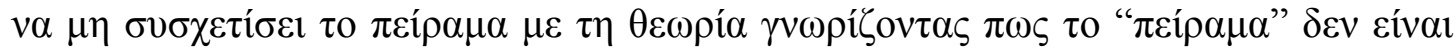

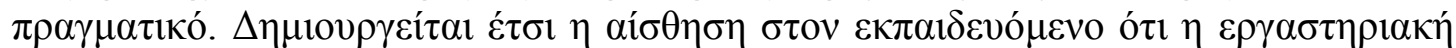

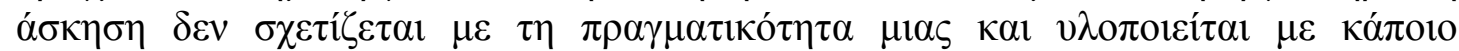

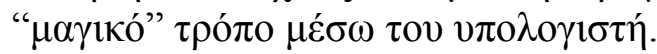

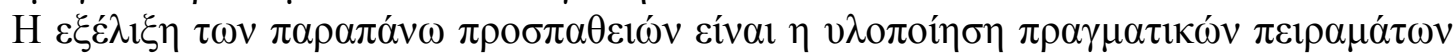

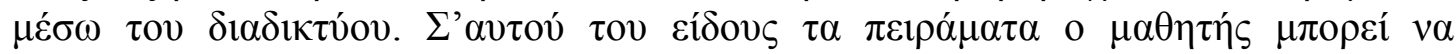

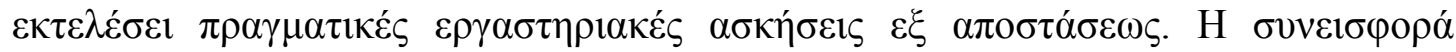

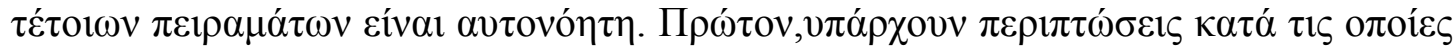

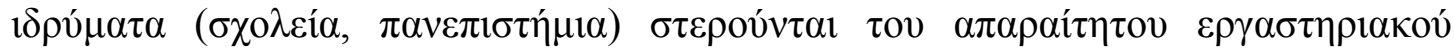

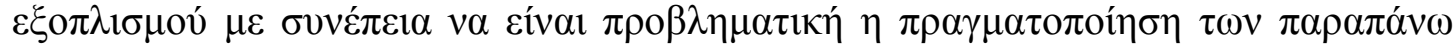

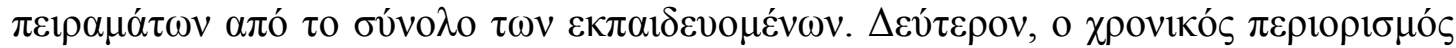

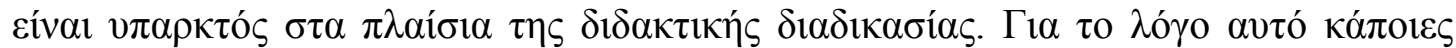

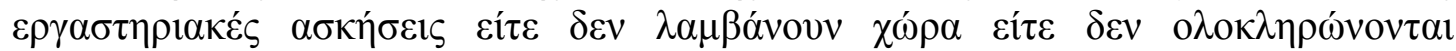

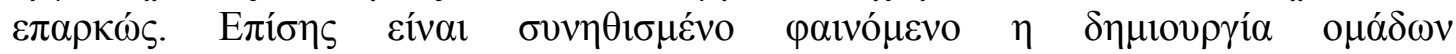

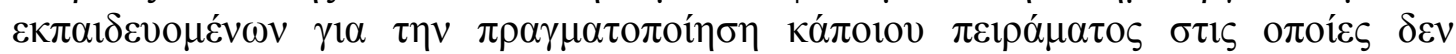

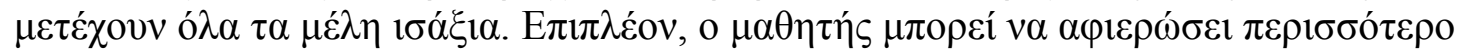

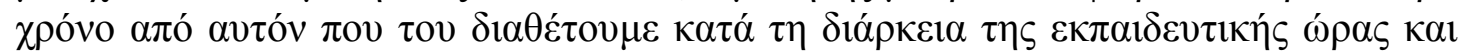

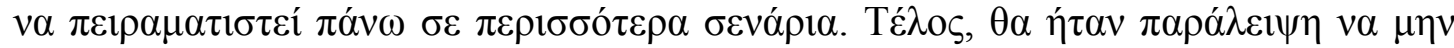

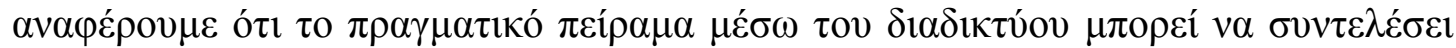

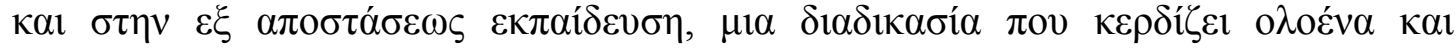

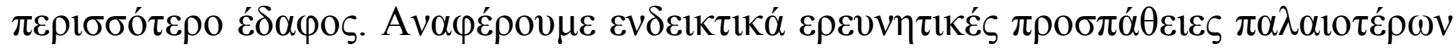

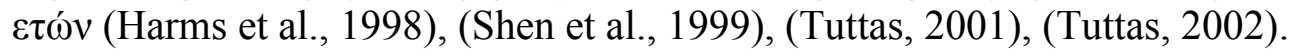

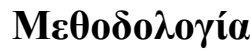

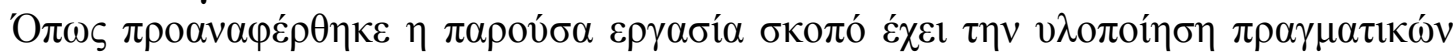

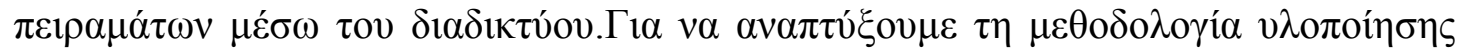

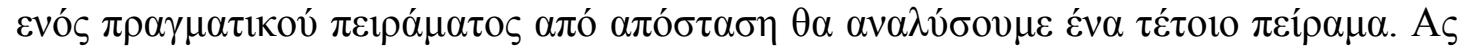

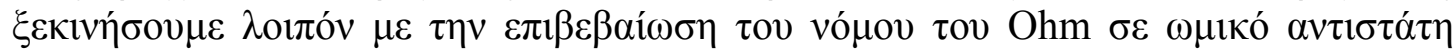

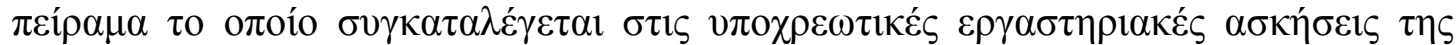

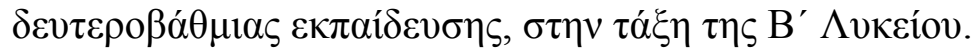

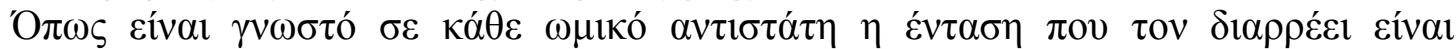

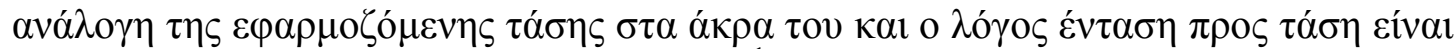

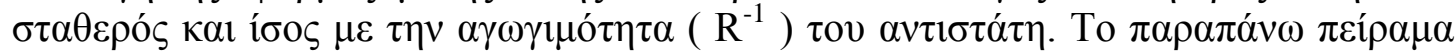

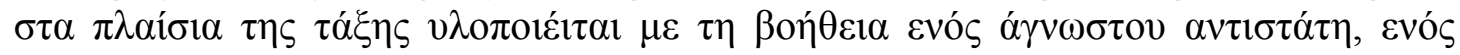

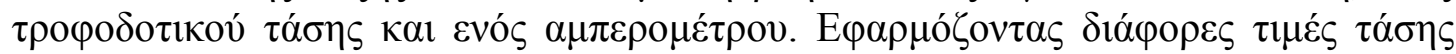

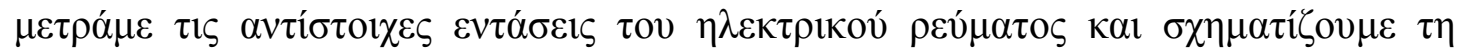

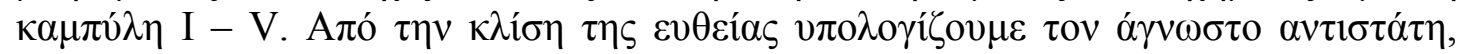

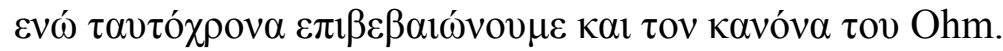

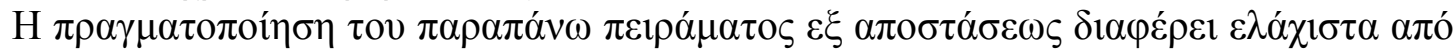

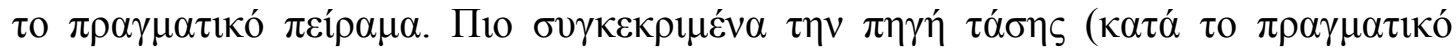

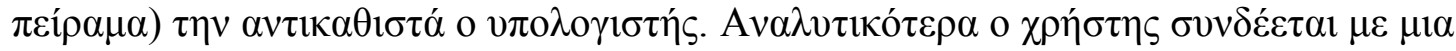




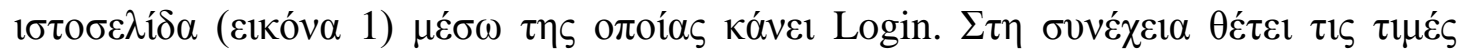

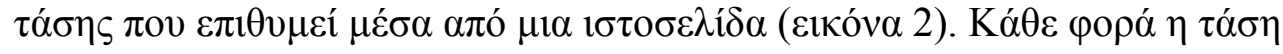

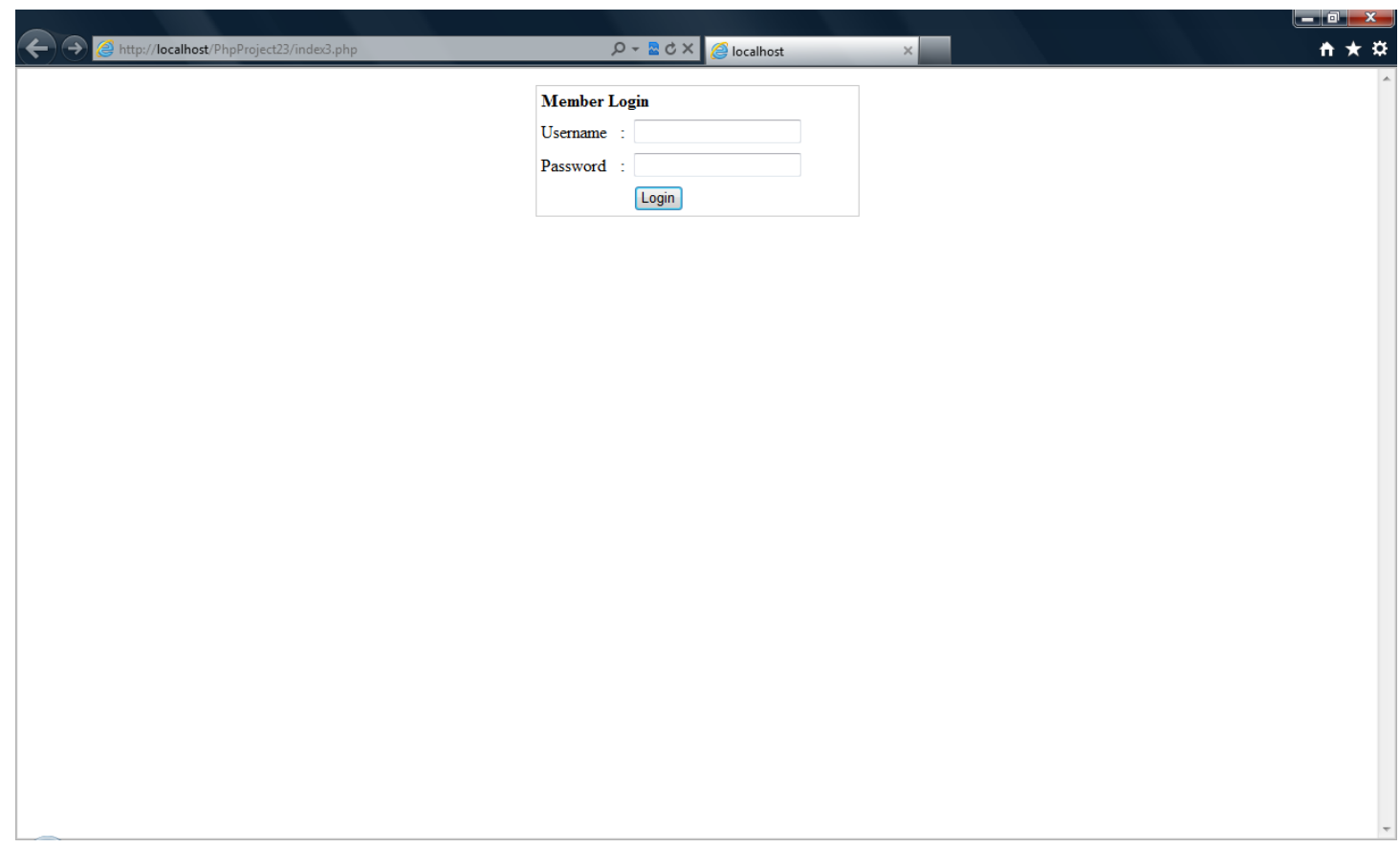

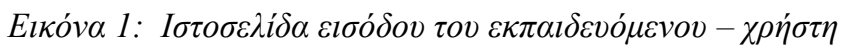

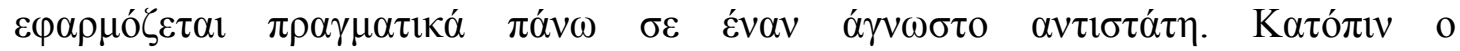

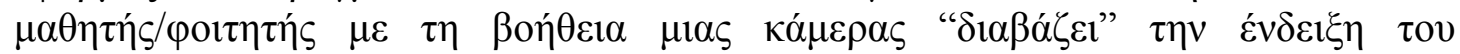

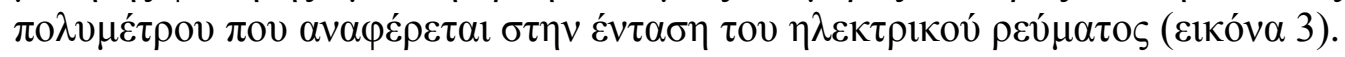

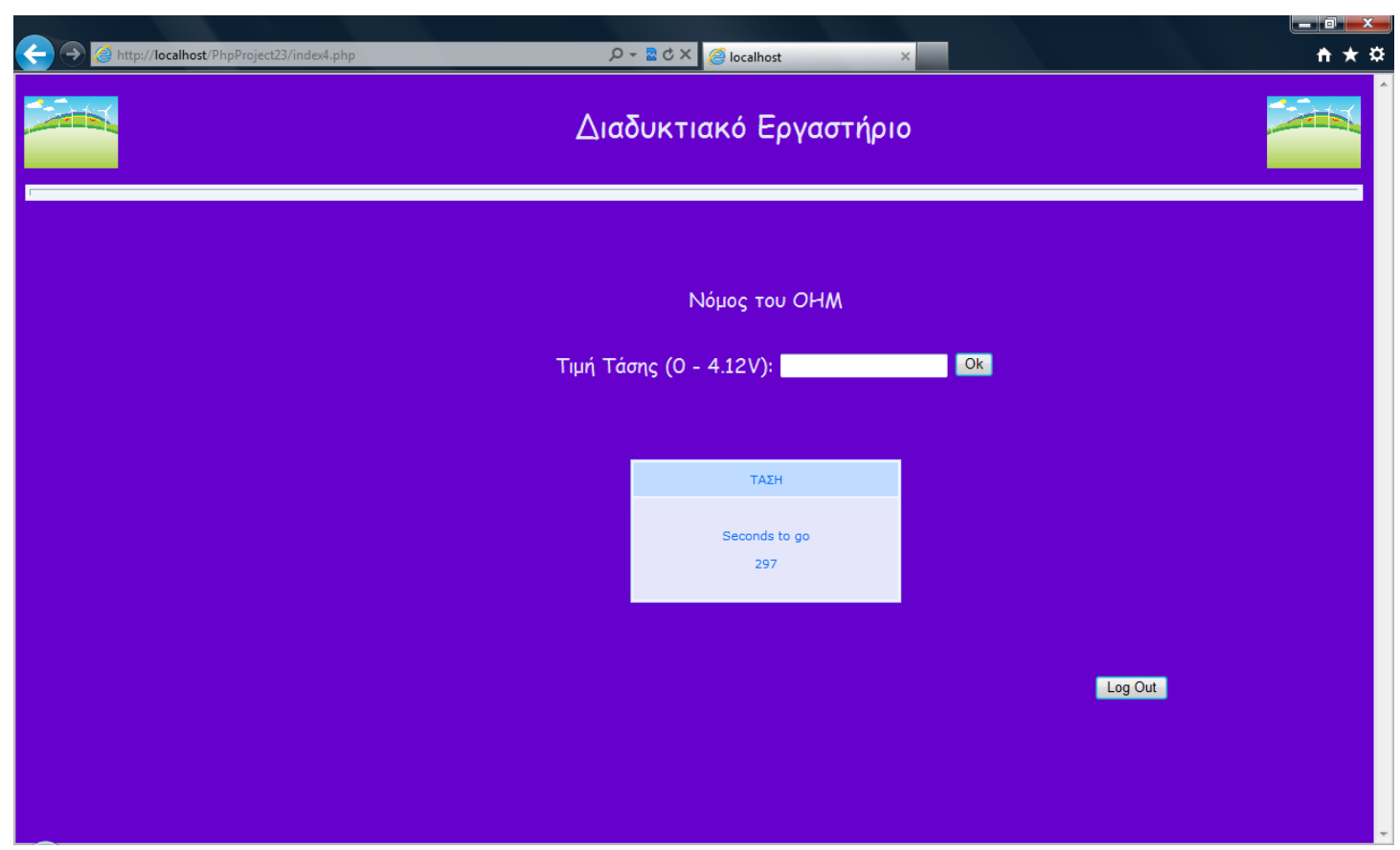

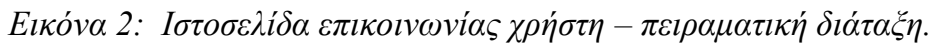




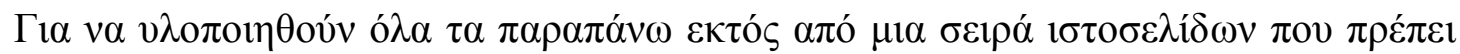

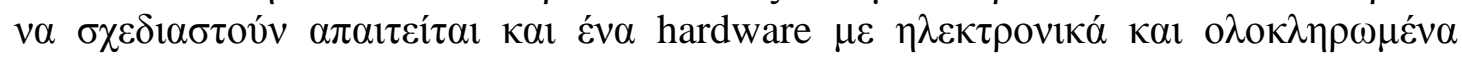

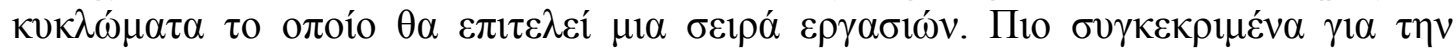

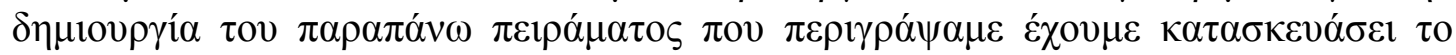

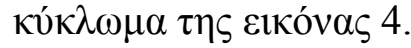

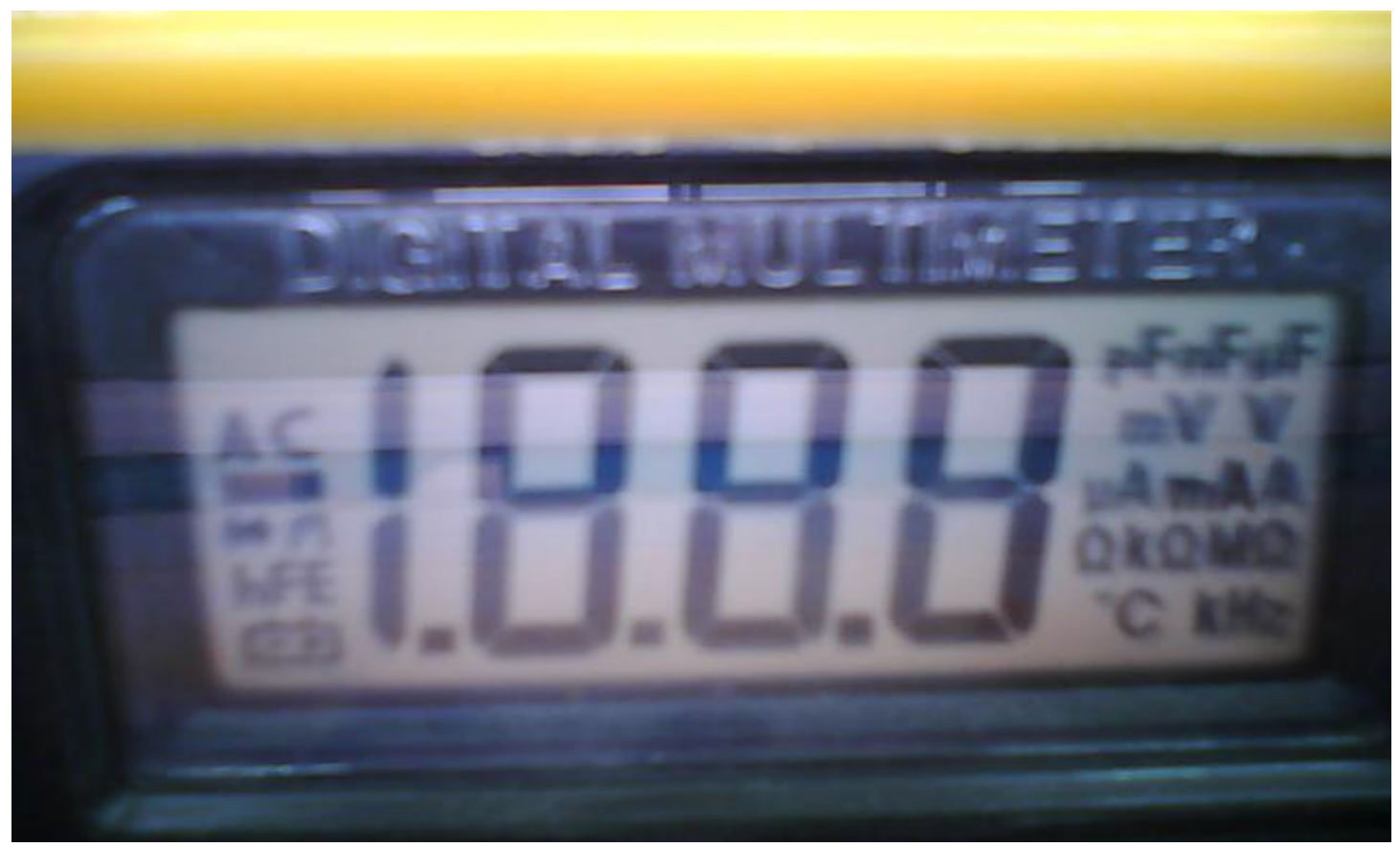

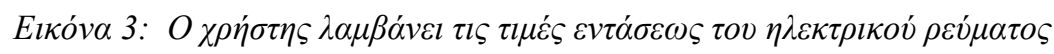

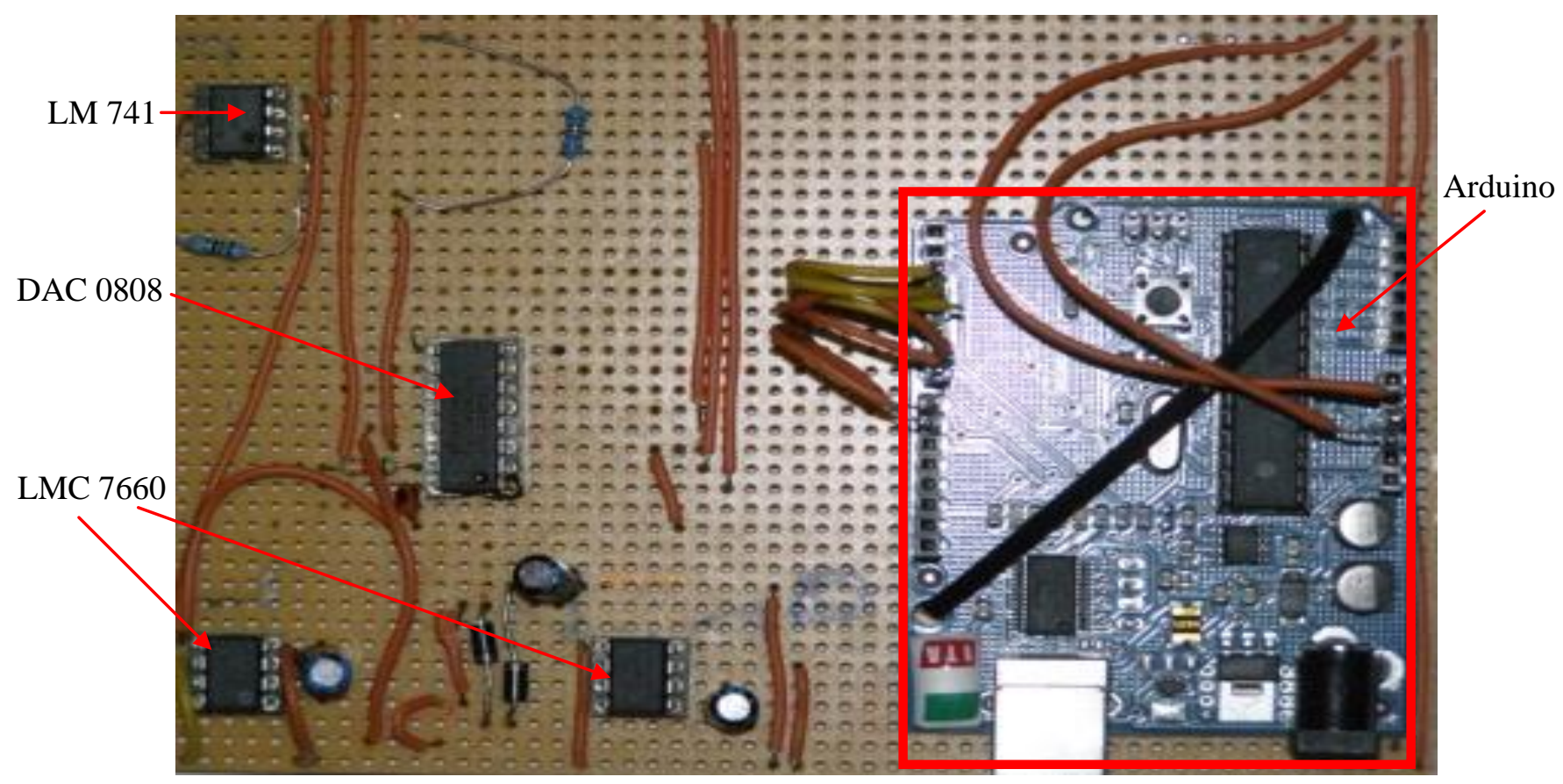

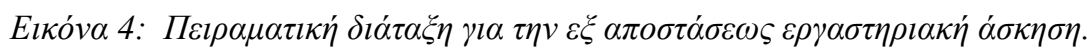

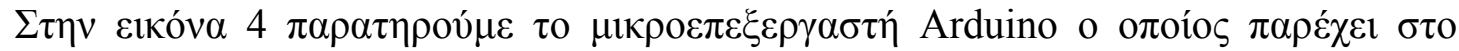

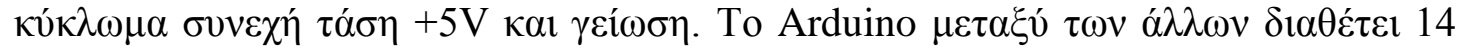

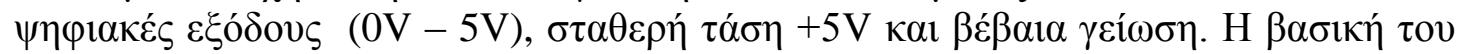

SECTION B: applications, experiences, good practices, descriptions and outlines, educational activities, issues for dialog and discussion 


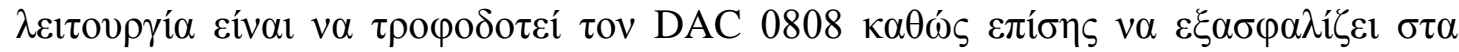

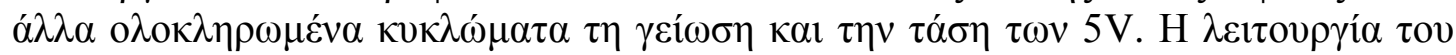

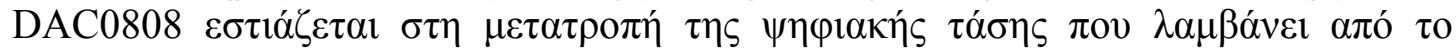

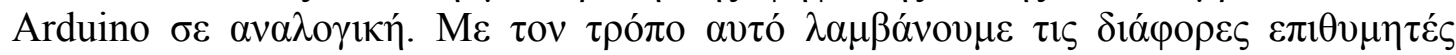

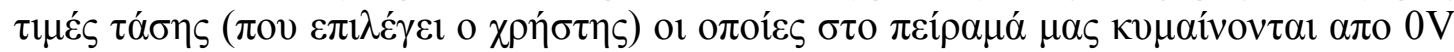

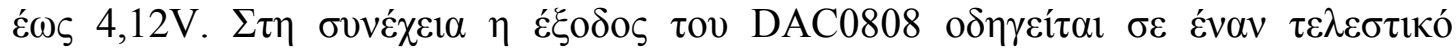

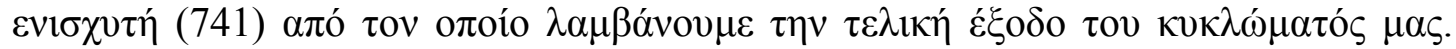

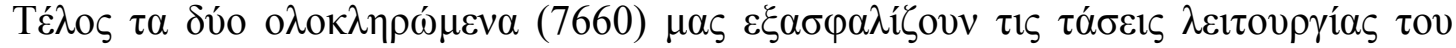

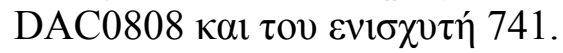

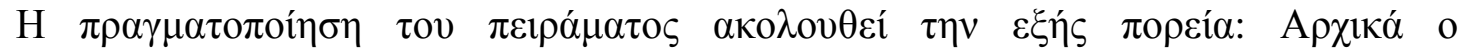

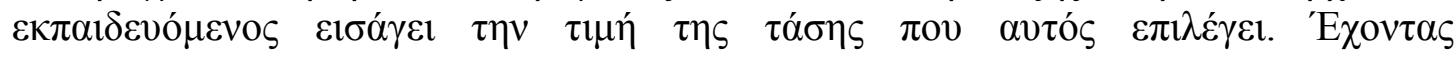

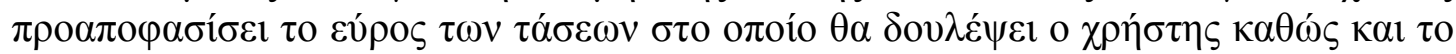

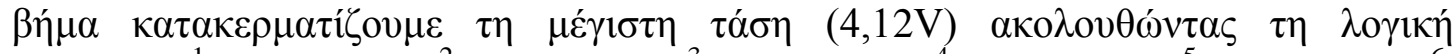
$\mathrm{A} 1=4,12 / 2^{1}, \quad \mathrm{~A} 2=4,12 / 2^{2}, \quad \mathrm{~A} 3=4,12 / 2^{3}, \quad \mathrm{~A} 4=4,12 / 2^{4}, \quad \mathrm{~A} 5=4,12 / 2^{5}, \quad \mathrm{~A} 6=4,12 / 2^{6}$,

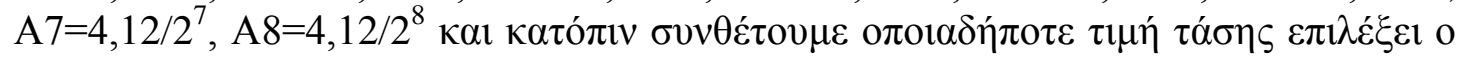

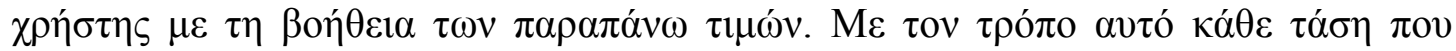

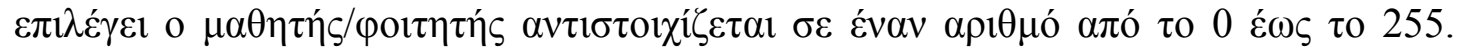

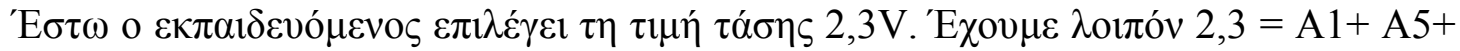

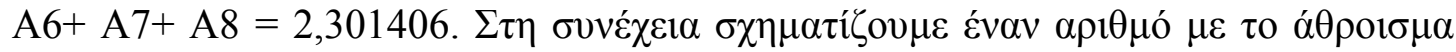

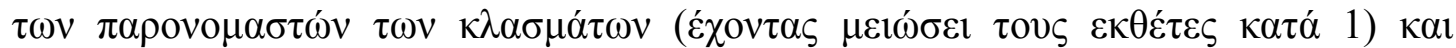

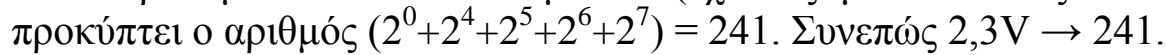

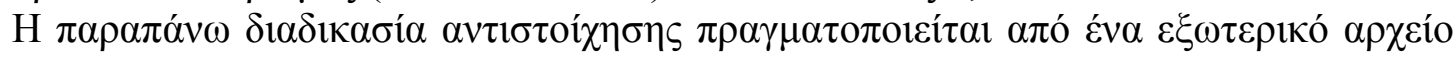

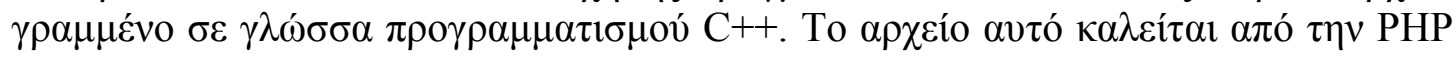

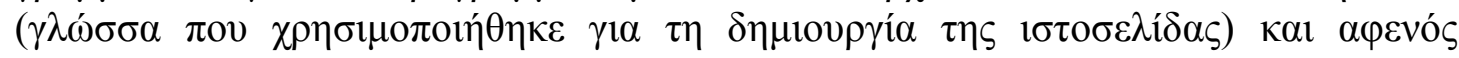

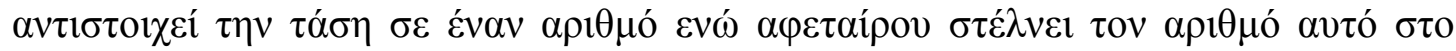

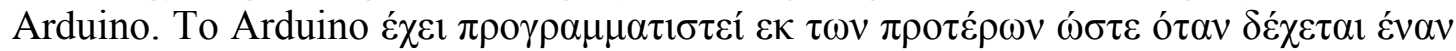

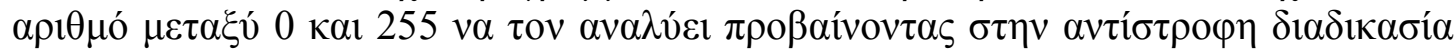

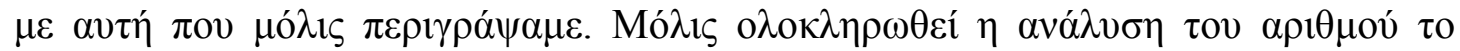

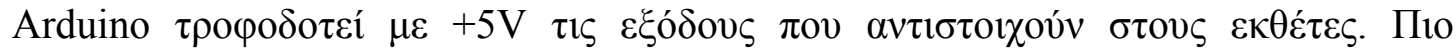

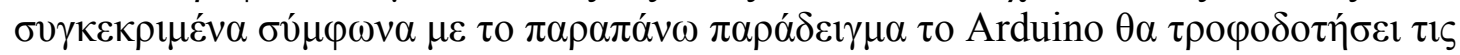

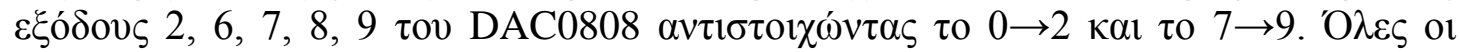

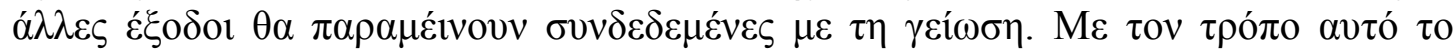

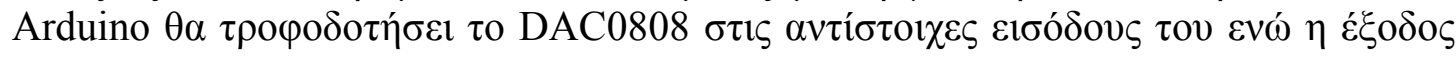

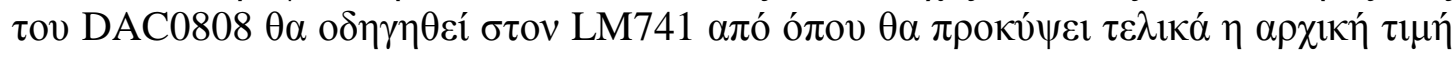

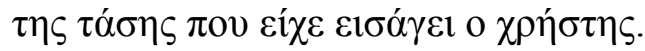

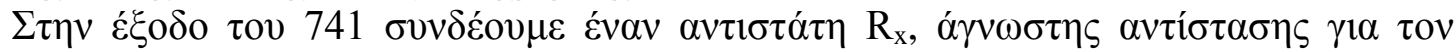

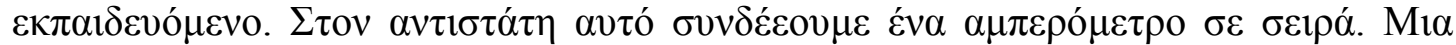

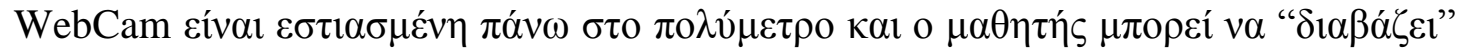

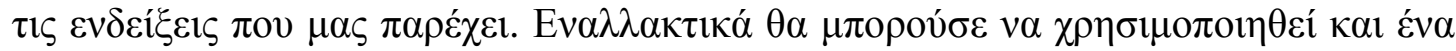

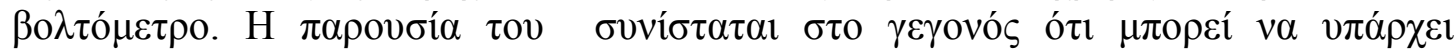

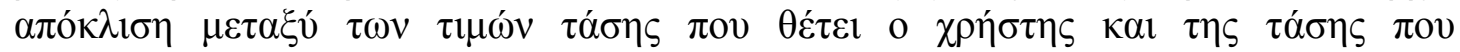

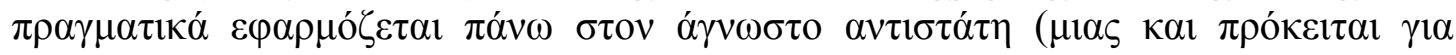

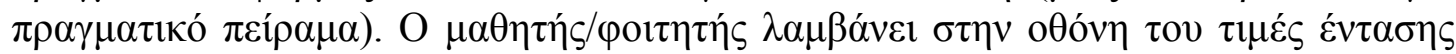

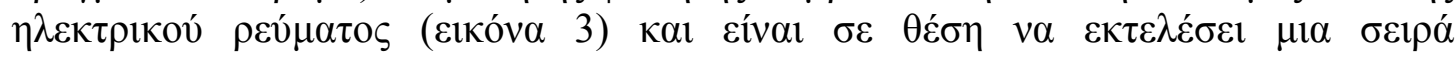

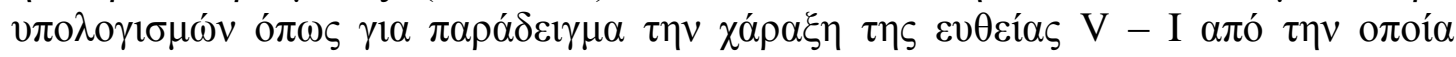

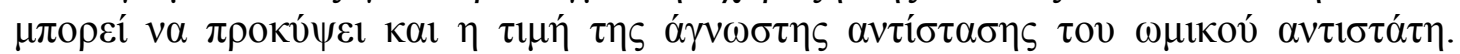

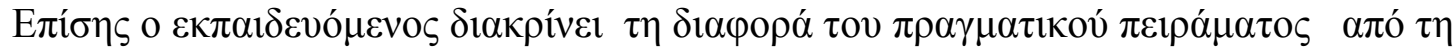

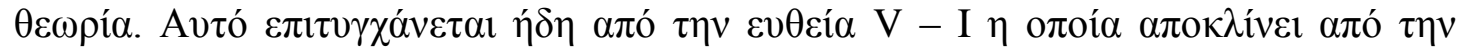

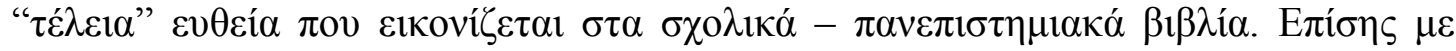

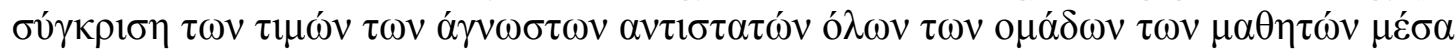




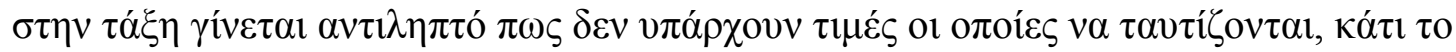

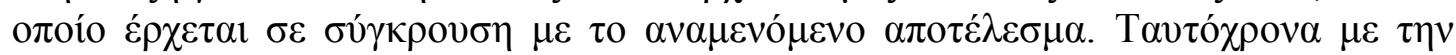

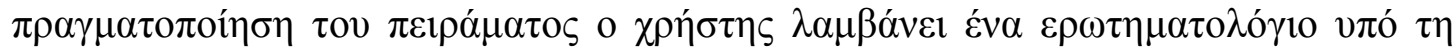

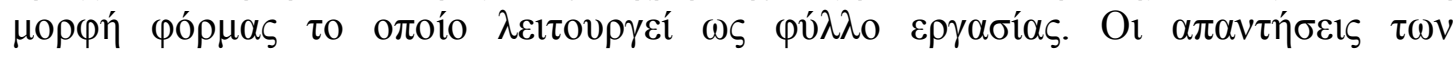
$\varepsilon \kappa \pi \alpha \imath \delta \varepsilon v o \mu \varepsilon \dot{v} \omega v$ a

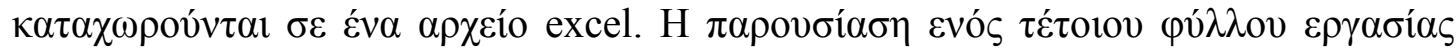

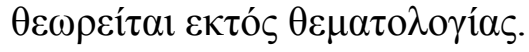

\section{$\Sigma v \mu \pi \varepsilon \rho \alpha ́ \sigma \mu \alpha \tau \alpha$}

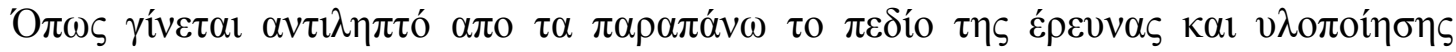

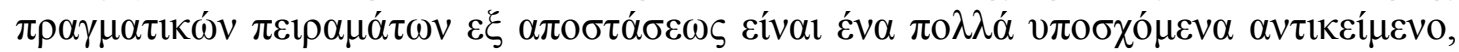

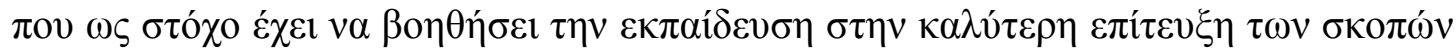

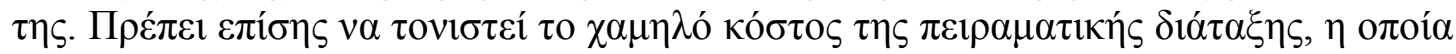

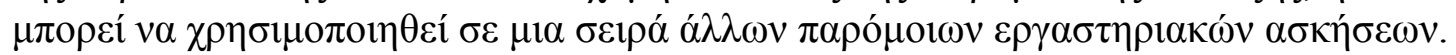

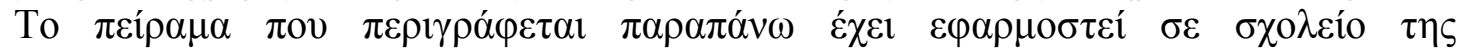

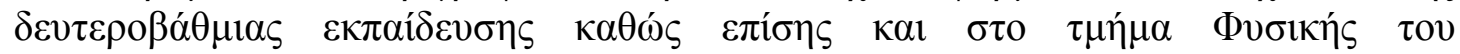

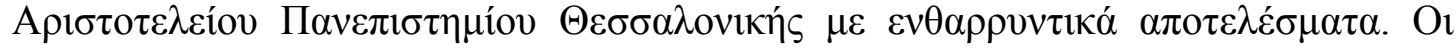

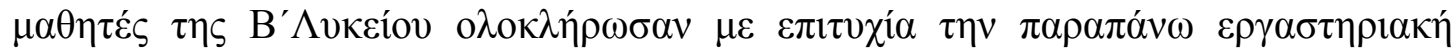

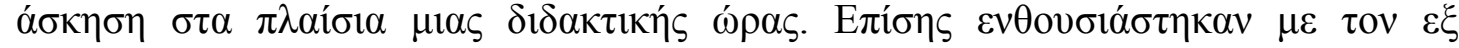

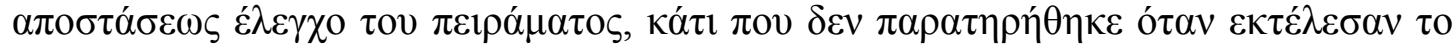

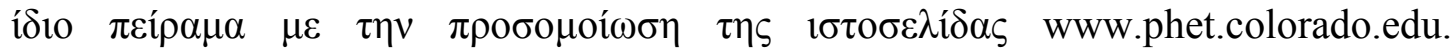

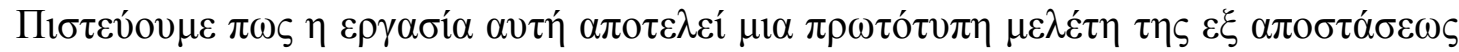

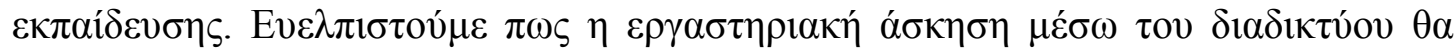

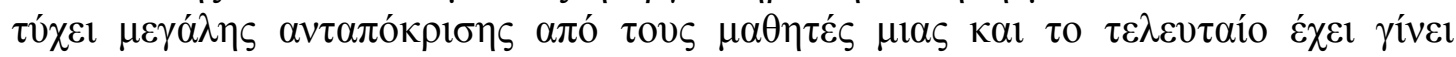

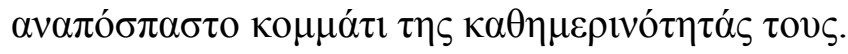

\section{Avapopés}

Harms, U. and Kurz, G., (1998). Virtual laboratory - an introductory unit 'POHL' storsional pendulum', Proceedings of the Third IEEE International Conference on Multimedia Engineering Education, \# 51, City University of Hongkong, China, Hongkong

Shen, H., Xu, Z., Dalager, B., Kristiansen, V., Strøm, Ø., Shur, M. S.,Fjeldly, T. A., Lu, J., Ytterdal, T., (1999). Conducting Laboratory Experiments over the Internet, IEEE Trans. on Education, Vol. 42, No. 3, pp. 180-185

Tuttas, J., Wagner, B., (2001). Distributed Online Laboratories, International Conference on Engineering Education (ICEE01), Oslo, Norway, August 6-10

Tuttas, J.,Wagner, B., (2002). The Relevance of Haptic Experience in Remote Experiments, World Conference on Educational Multimedia, Hypermedia \& Telecomunications (ED Media 2002)

Denver, USA, June 24-29, 2002 URL: www.arduino.cc 